\title{
CD99 type II is a determining factor for the differentiation of primitive neuroectodermal cells
}

\author{
Eun Jung Lee ${ }^{1}$, Hyun Gyu Lee ${ }^{1}$ \\ Sung Hye Park ${ }^{1}$, Eun Young Choi ${ }^{1}$ \\ and Seong Hoe Park \\ ${ }^{1}$ Department of Pathology \\ College of Medicine \\ Seoul National University, Seoul, Korea \\ ${ }^{2}$ Corresponding Author: Tel, 82-2-760-8266; \\ Fax, 82-2-763-6625; E-mail, pshoe@plaza.snu.ac.kr \\ Accepted 28 October 2003
}

Abbreviations: db-cAMP, $\mathrm{N}^{6}, 2^{2}$-Dibutyryl-cAMP; EFT, Ewing family of tumors; ES, Ewing's sarcoma cells; GFP, green fluorescent protein; PNET, primitive neuroectodermal tumor; RT-PCR, reverse transcription PCR

\begin{abstract}
CD99 is a $32-k D a$ cell surface molecule present on thymocytes, peripheral $T$ cells, many other hematopoietic stem cells and somatic cells were implicated in cell-cell adhesion and cell-activation phenomena. Two major subtypes have been identified so far, designated CD99 type I and type II. We have investigated the correlation between the degree of neural differentiation and the expression of CD99 subtypes in three differentially differentiated cell lines such as CADO-ES1, RD-ES, and SH-N-SY5Y, in order of differentiation. In addition, we induced differentiation of the RD-ES cell line by $N^{6}, 2^{\prime}$-dibutyryl-cAMP (db-cAMP). Six days after treatment with db-cAMP, RD-ES cell line has changed its morphology from uniform round cells to cells with neurites, and initially CD99 type Il-overexpressed RD-ES cells showed significant down-regulation of CD99 type II, whereas CD99 type I expression remained constant. When RD. ES cells were transfected with the cDNA encoding for CD99 type l-green fluorescence protein (GFP) and type II-GFP, CD99 type II transfected RD-ES cell line remained unchanged with morphology of undifferentiated form. Our data suggest that CD99 type II acts as a negative regulator in the neural differentiation of precursor cells that might occur during nerve system development.
\end{abstract}

Keywords: antigens, CD; antigens, differentiation; antigens, surface; cell differentiation; neurons; T-lymphocytes

\section{Introduction}

The cell surface glycoprotein CD99 is the product of the MIC2 gene that is located in the pseudoautosomal (pairing) region of the human $\mathrm{X}$ - and $\mathrm{Y}$-chromosomes (Goodfellow et al., 1986; Banting et al., 1989; Gil et al., 2002; Lee et al., 2002). The antigen is highly expressed on thymocytes, Ewing's sarcoma/primitive neuroectodermal tumor (ES/PNET) cells, pancreatic islet cells and leydig and Sertorli cells (Fellinger et al., 1991; Dworzak et al., 1994), and is expressed in other hematopoietic cells at moderate levels (Hahn et al., 1997). Originally, CD99 was described as a human thymus leukemia antigen, Ewing's sarcomaspecific membrane marker molecule, and a putative adhesion molecule (termed E2) involved in spontaneous rosette formation of $T$ cells with erythrocytes (Bernard et al., 1988; Aubrit et al., 1989; Gelin et al., 1989). In the studies using monoclonal antibodies (mAbs) against CD99 as stimulatory ligands, engagement of CD99 led to homotypic aggregation and death of thymocytes and Jurkat $T$ cells (Bernard et al., 1995; Bernard et al., 1997; Hahn et al., 1997; Pettersen et al., 2001) and induced up-regulation of $\mathrm{T}$ cell receptor (Waclavic et al., 1998) and major histocompatibility complex molecules on the cell surface (Choi et al., 1998; Kim et al., 2003).

Two types of CD99 molecules, a full-length protein (type I) and a short form (type II) harboring a deletion in the intracytoplasmic segment, are generated by alternative splicing (Ellis et al., 1994). Especially, the alternatively spliced form of CD99 transcripts (type II) are differentially expressed in a cell type specific manner among hematopoietic cells. Overexpression of the CD99 type II inhibited the homotypic aggregation of IM-9 cells by CD99 engagement (Hahn et al., 1997). Recently, it has been reported that the surface level of CD99 decreased when immature thymocytes were differentiated into mature $T$ cells (Bernard et al., 2002). In immature thymocytes, CD99 molecules are expressed on the cell surface as heterodimers of type I and type II and the decrease of surface level along the differentiation was due to the reduced expression of type II form in the mature T cells (Bernard et al., 2002). Namely, isomorphic expression of CD99 is 
tightly correlated with $\mathrm{T}$ cell differentiation.

ES cells have been described to represent the stage of either very early pluripotential precursor cells (neuronal stem cells) or PNET that can differentiate along rather heterogeneous group of cells including neuronal, glial, Schwannian, melanocytic, neuroendocrine pathway (Ewing et al., 1921; Cavassana et al., 1987; Moll et al., 1987). High expression of CD99 on ES/PNET cells is implicated as a diagnostic tool for the differential evaluation of small round cell tumor of childhood (Ambros et al., 1991). Similarly to thymocytes, the expression level of CD99 declines along the differentiation of neural cells from ES/PNET into neuroblostoma cells (Scotlandi et al., 1996) and only undifferentiated ES/PNET cells are susceptible to CD99induced apoptosis (Sohn et al., 1998).

The similarities of CD99 function and expression pattern between neural cells and $T$ cells suggested us that the decreased surface level of CD99 along the neural differentiation might be related to downregulation of CD99 type II antigen. In this study, we addressed this issue by comparing the amount of type II transcript of CD99 in immature RD-ES cells with that in the cells treated with db-cAMP to induce neural cell differentiation (Hara et al., 1991; Noguera et al., 1992; Kodama et al., 1994; Tetsuro et al., 1997) and checking the extent of db-cAMP-induced differentiation of the immature RD-ES cells that were forced to over-express CD99 type II.

\section{Materials and Methods}

\section{Cell culture}

Human ES cell lines, CADO-ES1 and RD-ES cell lines were obtained from the German Collection of Microorganism and Cell Cultures (Braunschweig, Germany) and the American Type Culture Collection (Rockville, MD), respectively. The human neuroblastoma cell line, SH-N-SY5Y, was generous gifts of Dr. Chong Jae Kim (College of Medicine, Seoul National University, Seoul, Korea). CADO-ES1 and RD-ES cell lines were maintained in RPMI 1640 (Jeil Biotechservices Inc., Daegu, Korea) supplemented with 15\% and 10\% FBS (Life Technology, Gaithersburg, MD), penicillin $(100 \mathrm{U} / \mathrm{ml})$ and streptomycin $(10 \mu \mathrm{g} / \mathrm{ml})$ at $37^{\circ} \mathrm{C}$ in $5 \% \mathrm{CO}_{2}$ in a humidified atmosphere. SH-NSY5Y cells were cultured in DMEM (Jeil Biotechservices Inc.) supplemented with 10\% FBS. For differentiation induction, RD-ES cells were cultured in RPMI 1640 supplemented with 10\% FBS in the presence of $\mathrm{N}^{6}-2^{1}$-dibutyryl-cAMP (db-cAMP) $(2.5 \mu \mathrm{M})$. The medium was changed every third day and maintained for up to 6 days. The morphological changes were evaluated under inverted microscope.

\section{Antibodies and reagents}

The following mAbs were used: anti-CD99 type I and II (FITC- or PE-conjugated YG32 for flow cytometric analysis, DiNonA, Seoul, Korea). db-cAMP was purchased from Sigma (St. Louis, MO). FuGENE6 was obtained from Roche Diagnostics Corporations.

\section{Immunofluorescent microscopy}

For immunofluorescent analysis, cells transfected with CD99 type I or CD99 type II were plated on glasscoverslips. After culture, CD99 type I-green fluorescent protein (GFP) and CD99 type II-GFP were detected by the GFP fluorescence under fluorescent microscope $(\times 600)$. To compare transfected cells with non-transfected cells, merged pictures were taken together.

RNA extraction, reverse transcriptase nested PCR (RT nested PCR) and semi-quantitative PCR So far, since no anti-CD99 antibody that can distinguish type II from type I isoform was developed, two isoforms of CD99 can be quantitative only by RT-PCR. We performed RT nested PCR by using primers specific for the CD99 type I and II transcripts to detect the expression of CD99 isoforms. Total RNA was isolated from the cells using an RNA extraction kit (Bioneer, Daejeon, Korea) and cDNA was first prepared by reverse transcription of $5 \mu \mathrm{g}$ RNA in a $20 \mu$ reaction volume containing PCR buffer, $1 \mathrm{mM}$ DTT, $200 \mu \mathrm{M}$ dNTPs, 200 pmole oligo-dT. The first round $\mathrm{PCR}$ was performed in $20 \mu$ reaction mix containing $20 \mathrm{mM}$ Tris- $\mathrm{HCl}(\mathrm{pH} 9.0), 1 \mathrm{U}$ Taq. DNA polymerase, $1 \mu \mathrm{M}$ each of the two primers, $250 \mu \mathrm{U}$ dNTPs, $40 \mathrm{mM} \mathrm{KCl}$, and $1.5 \mathrm{mM} \mathrm{MgCl}_{2}$ (Bioneer, Daejeon, Korea). After initial heating at $94^{\circ} \mathrm{C}$ for 5 min, 35 cycles of PCR were carried out $\left(94^{\circ} \mathrm{C}\right.$ for 30 $\mathrm{S}, 60^{\circ} \mathrm{C}$ for $30 \mathrm{~s}$ and $72^{\circ} \mathrm{C}$ for $30 \mathrm{~s}$ ). For the nested PCR, $1 \mu \mathrm{l}$ aliquot of the first-round PCR product was used as the template. The nested PCR was carried out in the same way with the first-round PCR. Primers were as follows: in order to amplify the CD99 type I form, outer primer 5'-ATGGCCCGCGGGGCTG-3', 5'-CTATTTCTCTAAAAGAGTACG-3', inner primer 5'GATGGTGGTTTCGATTTATC-3', 5'-CTCTTCCCCTTCTTTCCTGTGGCTGCC-3', and to amplify the CD99 type II form, outer primer 5'-ATGGCCCGCGGGGCTG3', 5'-CCCTAGGTCTTCAGCCAT-3', inner primer 5'GATGGTGGTTTCGATTTATC-3', 5'-CTCTTCCCCTTCTTTCCTGTGGCTGCC-3'. Dilution fold is indicated. We used $\beta$-actin as a positive control of the reaction.

Fluorescent staining and flow cytometric analysis Cells $\left(5 \times 10^{5}\right)$ were harvested and washed with PBS containing $1 \%$ bovine serum albumin and $0.1 \%$ 
sodium azide. And cells were incubated with antiCD99 mAb (FITC-conjugated YG32, $1 \mu \mathrm{g} / 100 \mu \mathrm{l}$ ) in PBS containing $1 \%$ BSA and $0.1 \%$ sodium azide for $1 \mathrm{~h}$ at $4^{\circ} \mathrm{C}$, flow cytometry analysis was performed on a FACScan (Becton Dickinson, San Jose, CA). To gate the viable cells, ES cells stained for surface expression of CD99 were washed, suspended in 400 $\mu \mathrm{l}$ of $1 \%$ BSA1 PBS containing $10 \mu \mathrm{g} / \mathrm{ml}$ of propidium iodide (PI; Sigma, St. Louis, MO).

\section{Gene construction and stable transfection}

To further analyze the functional consequences of the distinct CD99 isoform expression in RD-ES cells, we performed transfection experiments. A panel of stably transfected cells was prepared using the expression vector pEGFP-N1 (Invitrogen, Groningen, Netherlands), conferring resistance to G418 antibiotics (Sigma). The unspliced CD99 (type I) cDNA and the spliced CD99 (type II) were inserted into the HindIII/Kpnl cloning site. Correct nucleotide sequence was confirmed by sequencing. The recombinant vectors were transfected into RD-ES cells using FuGENE 6 reagent (Roche). Establishment and subcloning of stable cell lines were accomplished by culturing transfectants in the presence of $0.5 \mathrm{mg} / \mathrm{ml}$ of G418 (Sigma) for 1 month.

\section{Results}

Expression level of CD99 is inversely correlated to the level of neural cell differentiation

The fact that CD99-induced apoptosis occurs only in undifferentiated ES cells, not in differentiated ES cells (Sohn et al., 1998), led us to ask whether the re-

A

CADO-ES1

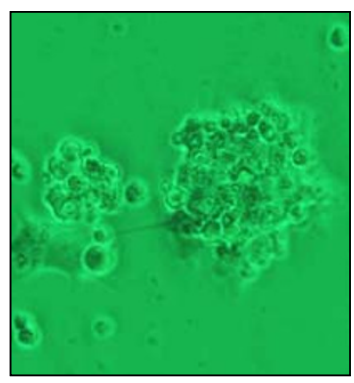

RD-ES

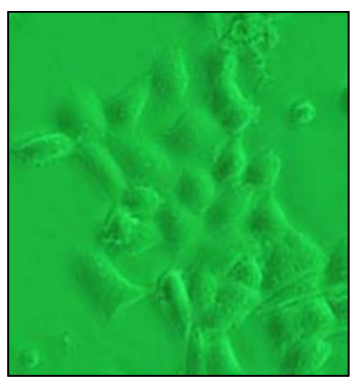

SH-N-SY5Y

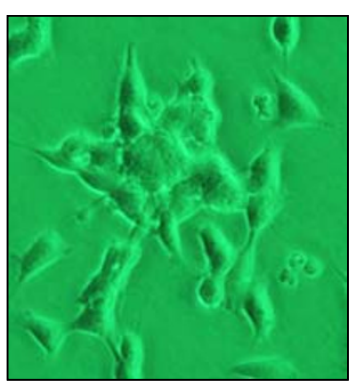

B
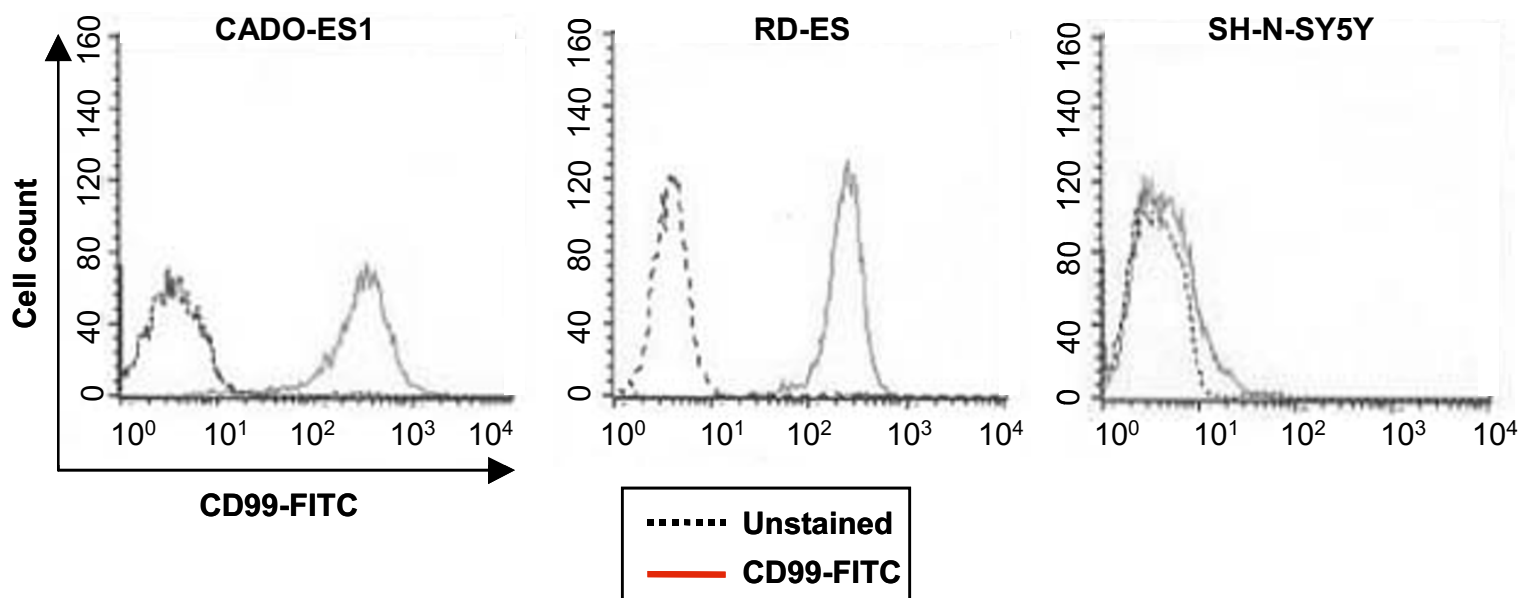

Figure 1. The surface expression pattern of CD99 in Ewing's sarcoma and neuroblastoma cells. CD99 expression in respective cell lines according to their differentiation status. (A) CADO-ES1, RD-ES and SH-N-SY5Y cells were cultured in normal status. The images show the CADO-ES1, RD-ES, SH-N-SY5Y cells. (B) Live cells were gated and analyzed on a FACScan flow cytometry. Sorting profiles were plotted according to fluorescence intensity for CD99-FITC. Left peaks represent baseline fluorescence for the negative population of unstained cells (dotted line). Ewing's sarcoma cell lines highly express CD99 but SH-N-SY5Y cells express CD99 of small amount. 
sistance of differentiated ES cells to CD99-induced apoptosis would be related to the expression level of CD99 along the differentiation of the neural cells, especially CD99 type II, as was the case for T cells (Bernard et al., 1997). To test this, first, we checked the expression level of CD99 in three different tumor cell lines of neural origin, CADO-ES1, RD-ES and SH-N-SY5Y that are supposed to differ in their neurogenic potentials, with neuroblastoma cells (SH-N-SY5Y) considered as the most differentiated tumor cell line. They showed different degrees of neurite formation, with increased neurite formation in the order of CADOES1, RD-ES and SH-N-SY5Y (Figure 1A). When the three cell lines were analyzed for the expression of CD99 with flow cytometry, the expression levels of CD99 on the three cell lines inversely correlated with the differentiation status of the cell lines, with highest expression on CADO-ES1 and almost negligible ex- pression on SH-N-SY5Y (Figure 1B).

We choose RD-ES cells to compare CD99 expression level between before and after differentiation, which show a little neurite formation in culture with intermediately differentiated morphology and are relatively easy to induce differentiation by db-cAMP treatment (Noguera et al., 1992; Kodama et al., 1994; Tetsuro et al., 1997; Sohn et al., 1998). Treatment of db-cAMP to RD-ES cell line decreased cell proliferation and induced extensive neurite-like branches (Figure 2A). Thus db-cAMP treated cells could be clearly distinguished from undifferentiated cells of small and round shape. On flow cytometry analysis, the surface expression level of CD99 was dramatically decreased in the differentiated RD-ES cells (Figure 2B), supporting that CD99 expression level was inversely correlated with the differentiation status of neural cell lineage (Figure 1B).
A

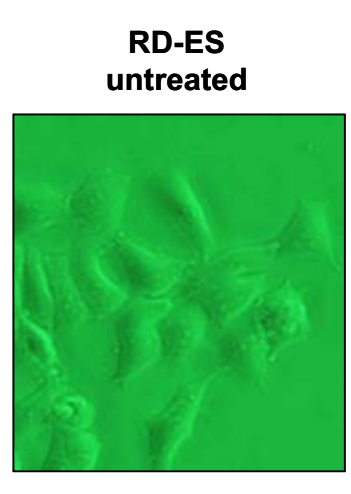

B

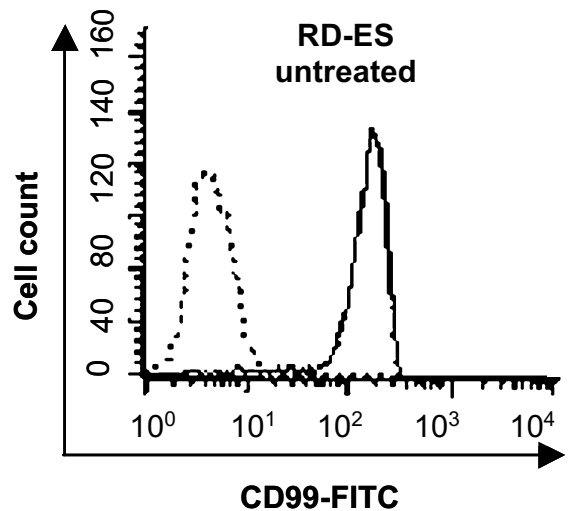

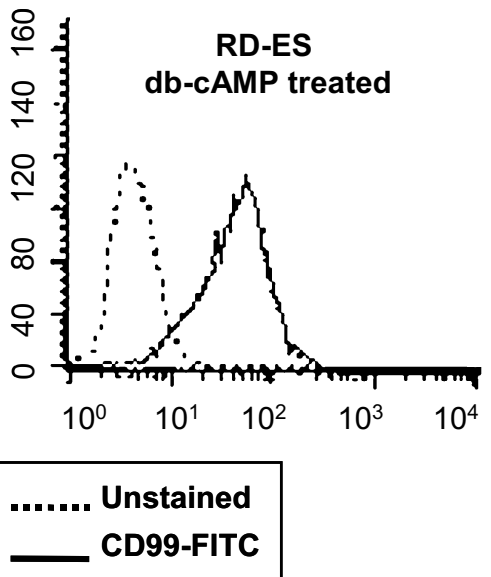

Figure 2. The down-regulation of CD99 in the differentiated RD-ES cells. The effect of db-cAMP on the expression of CD99. (A) RD-ES cells were cultured in the presence or absence of db-cAMP $(2.5 \mu \mathrm{M} / \mathrm{ml})$ for 6 days. The images show the RD-ES and differentiated RD-ES cells. (B) Live cells were gated and analyzed on a FACScan flow cytometry. Sorting profiles were plotted according to fluorescence intensity for CD99-FITC. Left peaks represent baseline fluorescence for the negative population of unstained cells (dotted line). The expression level of CD99 was markedly reduced in the db-cAMP-treated RD-ES cells (right panel) as compared with the undifferentiated RD-ES cells (left panel). 


\section{CD99 type II is responsible for the reduced surface} expression of CD99 on differentiated ES cells

Next, we asked which type of CD99, type I or type II was down regulated according to neural cell differentiation. Since type II molecules overlap with unprocessed type I molecules in western blot, RT-PCR for each type of CD99 molecules was performed to detect each transcript in the cell lines corresponding to different differentiation status, CADO-ES1, RD-ES, db-cAMP treated RD-ES, and SH-N-SY5Y. While RTPCR products of CD99 type I were detected in all four cell lines with almost same band intensity, the bands of RT-PCR product of CD99 type II varied according to the cell differentiation (Figure $3 \mathrm{~A}$ ). No band or very weak band of RT-PCR product of CD99 type II was obtained from the most differentiated cell line SH-N-SY5Y or db-cAMP treated RD-ES, respectively, in contrast to the bands from CADO-ES1 and RD-ES, with 2-3 fold increased intensity in the latter two cells (Figure 3B).The reduction of CD99 type II expression along the maturation was quantified with semiquantitative RT-PCR using the RD-ES cells. According to the band intensities of and densitometric measurements of the PCR products, it was revealed that the amount of type II transcripts from the cells treated with db-cAMP was 9-27 fold lower than that from the cells maintained undifferentiated (Figure $3 \mathrm{C}$ ). This change in transcript level of CD99 type II was in contrast to the steady level of type I transcript along the cell maturation under the range of dilutions in our experiment. These results indicate that the downregulation of CD99 expression on the differentiated cells was mainly due to reduced transcription of CD99 type II molecules along the differentiation of the neural cells.
A

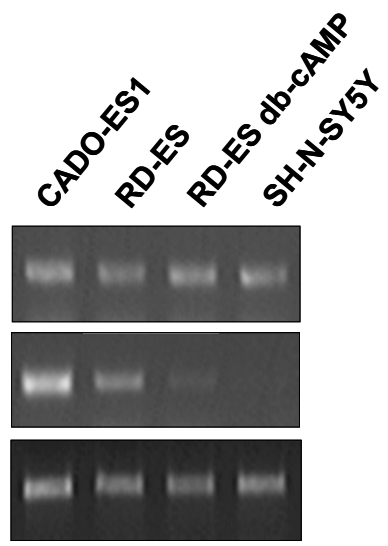

C

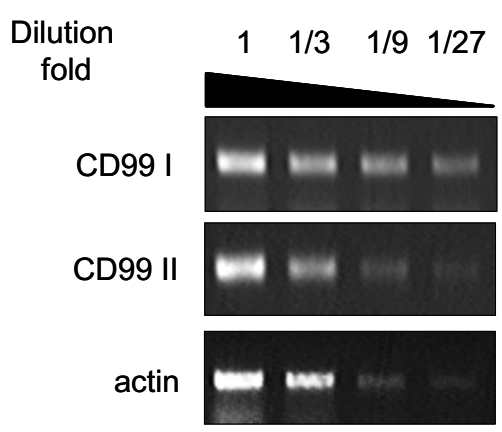

RD-ES
B

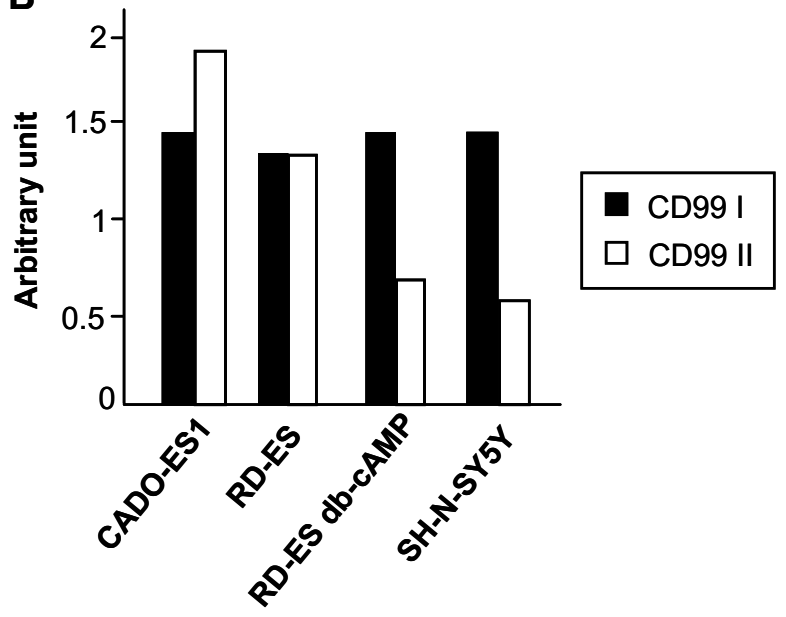

$\begin{array}{llll}1 & 1 / 3 & 1 / 9 & 1 / 27\end{array}$

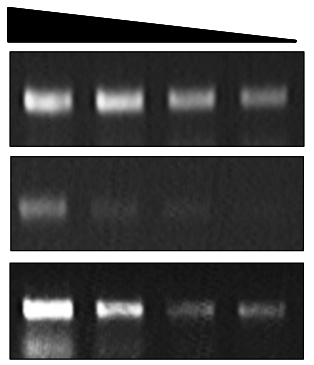

RD-ES db-cAMP

Figure 3. The down-regulated isotype of CD99 in the differentiated RD-ES cells is type II form. RD-ES cells were cultured in the presence or absence of db-CAMP $(2.5 \mu \mathrm{M})$ for 6 days and CADO-ES1, SH-N-SY5Y cells were cultured in normal status. The expression pattern of CD99 type I and type II form mRNA was observed by semiquantitative reverse transcriptase-nested-polymer chain reaction (RT nested PCR) using the specific primer pairs predicted to amplify the type I or II isoform of CD99 mRNA (A). (B) The quantification mRNA levels of CD99 I and II are shown. (C) Semiquantitative PCR are performed and serial dilution fold is indicated. The amount of mRNA in samples was normalized by PCR of human $\beta$-actin. 


\section{CD99 type II overexpressed RD-ES cells maintained CD99 expression after db-cAMP treatment}

Based on the results that reduced transcription of CD99 type II was responsible for the reduced surface expression of CD99 on differentiated cells, db-cAMPtreated RD-ES and SH-N-SY5Y, we questioned whether sustained high expression of CD99 type II could make undifferentiated RD-ES cells resistant to $\mathrm{db}$ cAMP-induced differentiation. To address this issue, we introduced RD-ES cells with a plasmid containing CD99 type II gene of which cytoplasmic domain was fused with GFP to distinguish it from endogenous CD99, and then the cells were treated with $\mathrm{db}$-CAMP. Since the expression of the exogenous CD99-GFP molecule was driven by CMV promoter, we checked whether high level of CD99 could be maintained on the surface of the CD99 type II-GFP transfected cells irregardless of internal gene control program following cell differentiation after db-cAMP treatment with flow cytometric analysis. When RD-ES cells transfected with a plasmid encoding for GFP molecule only were induced to differentiate with treatment of db-cAMP for 6 days, both populations of GFP-negative and -positive RD-ES cells showed down-regulation of CD99 molecules (Figure 4A). However, in the case of RD-ES cells transfected with a plasmid encoding CD99 type II-GFP, only GFP-negative population down-regulated CD99 after db-cAMP treatment with GFP-positive population maintaining high level of CD99 on the cell surface (Figure 4B). These results confirmed that the ectopic expression of CD99-GFP caused maintenance of CD99 expression at a high level independently of endogenous program of gene expression control re-
A

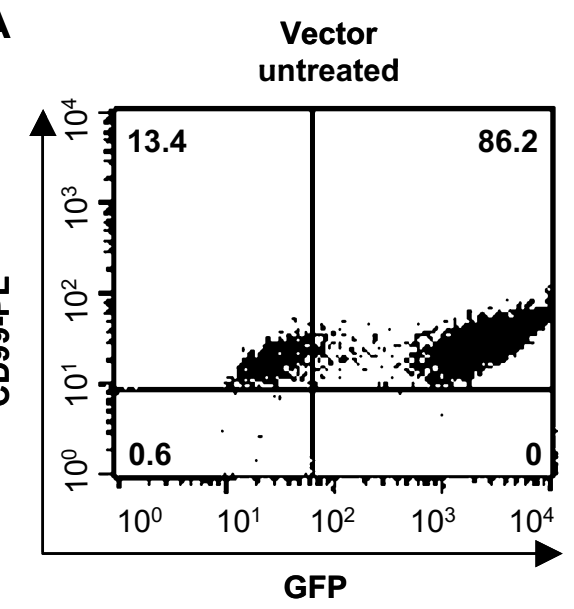

B

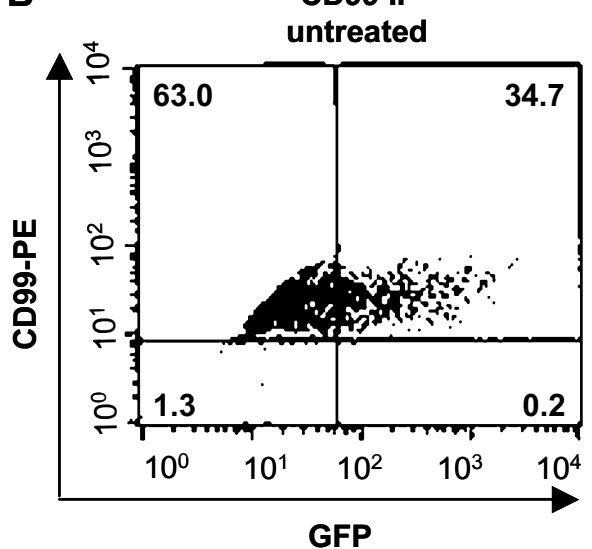

Vector

db-cAMP treated
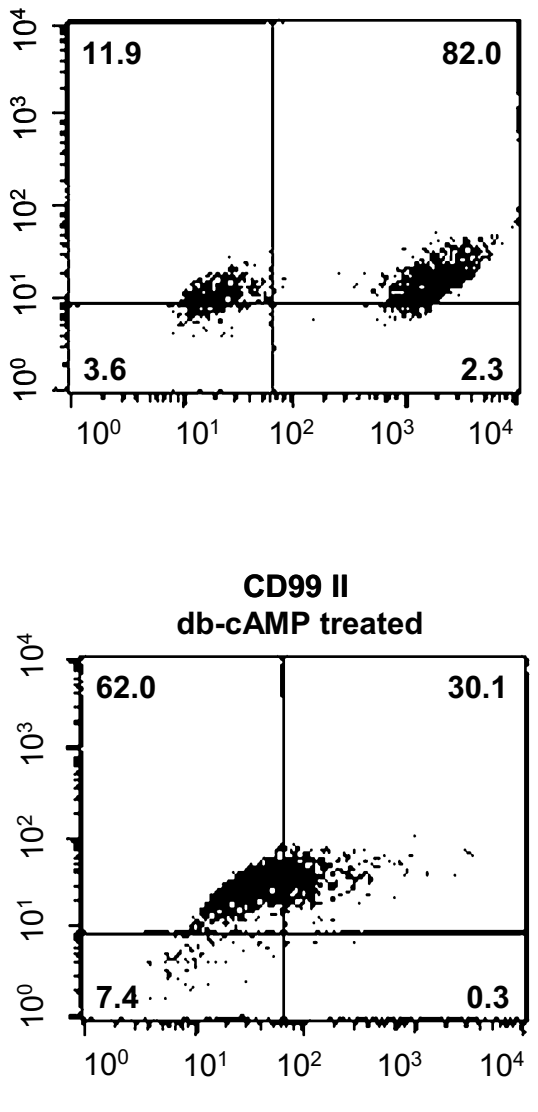

Figure 4. CD99 type II overexpressed RD-ES cells sustained surface expression of CD99 after db-CAMP treatment. CD99 type II overexpressed RD-ES cells were cultured in the presence or absence of db-cAMP $(2.5 \mu \mathrm{M} / \mathrm{ml})$ for 6 days. Live cells were gated and analyzed on a FACScan flow cytometry. Number of each corner represents cell percentage of the gated region. Sorting profiles were plotted according to fluorescence intensity for CD99-GFP and CD99-PE. CD99 type Il-overexpressed cells sustained the CD99 expression after db-CAMP treatment (B) as compared with the vector-transfected cells (A). Data shown are representatives of three independent experiments. 
A

\section{RD-ES untreated}
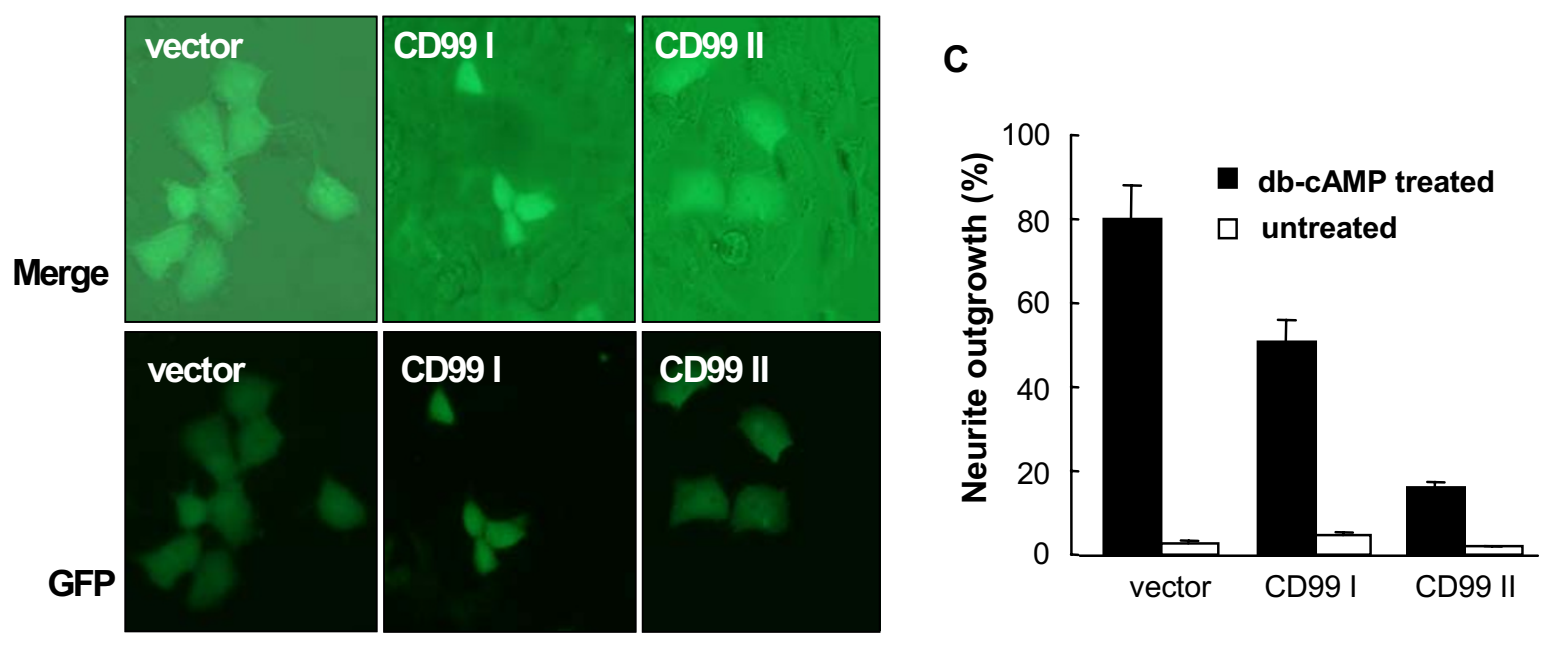

B

RD-ES db-cAMP treated

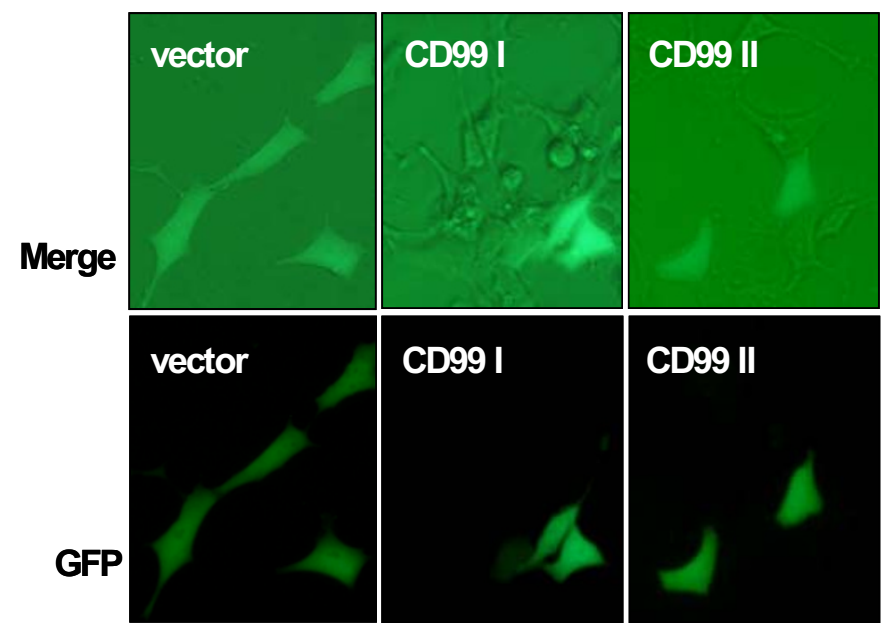

Figure 5. Overexpression of CD99 type $\|$ in RD-ES cells decreased elongated neurite expansion of differentiated RD-ES cells. The function of CD99 type II in differentiated RD-ES cells. CD99 type I or II transfected RD-ES cells were cultured in the absence (A) or presence of db-cAMP $(2.5 \mu \mathrm{M} / \mathrm{ml}, \mathrm{B})$ for 6 days and cells were plated on glass coverslips. GFP-vector, CD99 type I-GFP and CD99 type II-GFP were detected by the GFP fluorescence. Slides were visualized by fluorescent microscope magnification of $X 600$ (Nikon). The green fluorescence is shown on the lower panel side pictures and the merged pictures on the upper panel. Representative results are shown from three independent experiments. (C) Quantification of at least 10 different fields at each case from three independent experiments.

lated to differentiation.

Next, we investigated morphological change of CD99-transfected RD-ES cells, which accompanies neural differentiation, to test whether the maintenance of high level of CD99 rendered the cells to be resistant to differentiation induction by treatment of $\mathrm{db}$ cAMP for 6 days: Undifferentiated cells show homogeneous small round appearance, while differentiated cells consist of irregular cell population, exhibiting a neuronal cell like appearance with abundant neuritis formation. The RD-ES cells that expressed exogenous
CD99 molecules were easily distinguished from nontransfected cells by the GFP expression under fluorescent microscope. Both of the cells transfected with GFP and with CD99 type II-GFP constructs showed small round appearance before any treatment as did the surrounding untransfected cells (Figure 5A). After the induction to differentiate, 10 different fields of the transfected cells under fluorescent microscope were checked for the cell appearance and GFP expression. Approximately $84 \%$ of the type II-GFP-transfected cells remained as undifferentiated, maintaining small 
round shape whereas, the majority of the cells transfected only with GFP construct showed differentiation morphology with the formation of neurite outgrow (Figure $5 \mathrm{~B}$ ) as much as did untransfected cells surrounding type II-GFP transfected cells. These results clearly demonstrate that overexpression of CD99 type II led to maintenance of CD99 at high levels on the cell surface and the high expression CD99 molecules kept the cells in undifferentiated status, implying that CD99 type II is a determining factor in RD-ES cell differentiation.

\section{Discussion}

In this report, we demonstrated that two isoforms of CD99 were differentially related to the differentiation of the Ewing's sarcoma cell lines. Treatment of $\mathrm{db}$ CAMP to the RD-ES cell line induced a decrease of proliferation and formation of extensive branching of neurite-like processes, a similar cell morphology to those produced by sympathetic neurons in primary cell cultures (Noguera et al., 1992; Kodama et al., 1994; Tetsuro et al., 1997; Sohn et al., 1998). Such a differentiation of RD-ES cells in vitro led to significant selective down-regulation of CD99 type II, despite of sustained expression of type I. Most importantly, the cells forced to maintain CD99 type II at a high level remained undifferentiated form after db-cAMP treatment, keeping surface expression of CD99 at a high level independently of endogenous gene regulation.

Differential roles of CD99 isoforms have been studied in thymocytes (Alberti et al., 2002). Bernard and Alberti have demonstrated that the expression pattern of the two CD99 isoforms depends on the differentiation status of T cells (Alberti et al., 2002). The expression of CD99 decreased when immature thymocytes were differentiated into mature $T$ cells and this was due to reduced expression of type II form. The single expression (transfection) of type $\mathrm{I}$ isoform in CD99 deficient Jurkat $T$ cells could lead to adhesion events, whereas expression of the heterodimeric forms of CD99 composed of type I and type II induced the apoptosis (Alberti et al., 2002). Therefore it is likely that expression of CD99 type II is universally related to immaturity of cells in both neural and hematopoietic lineages. And surface level of CD99 relies more on the amount of type II than that of type I molecule. In immunohistochemical studies of normal human brain, CD99 is expressed highly on ependymoma where immature and undifferentiated precursor cells reside (Choi et al., 2001). The inverse relationship between the amount of type II CD99 mRNA and differentiation of neural cells suggests that CD99 type II might be expressed highly on the cells in ependymoma of brains, and reduced apoptosis of cortical thymocytes from anencephaly after CD99 engagement (Shin et al., 1999) might be related to the amount of type II transcripts in the cells.

In terms of the application of CD99 to immunotherapeutic trials, it has been reported that CD99 may be used as an immunotherapeutic tool for the treatment of Ewing's Family of Tumors (EFT) patients (Sohn et al., 1998; Scotland et al., 2000). Engagement of CD99 significantly inhibited the in vivo and in vitro growth ability of Ewing's tumor cells by delivering an apoptotic stimulus and reducing the malignant potential of these cells. And this ability of CD99 was strictly dependent on the level of expression of CD99, requiring the presence of a sufficient number of these molecules on the surface expression of EFT cells. They suggested the potential therapeutic value of CD99 as a specific target for innovative treatment of EFT patients. In this respect, regulation of type II molecules might be a target for development of treating agent for Ewing's tumor cells, avoiding any harm on surrounding intact differentiated neuronal cells. Understanding the exact mechanism for the regulation of CD99 type II will be provide a basis for the clinical application of this molecule.

In conclusions, our data indicates that CD99 type II may play a crucial role as a negative regulator in the ES cell differentiation. This may have two relevant implications. From a biological point of view, our results raise new questions on the function of CD99 molecule. Together with a previous study on $\mathrm{T}$ cells (Alberti et al., 2002), our data further supports the view of CD99 as a primitive marker associated with an inhibitory differentiation potential. From a clinical point of view, our data show the possibility of CD99 as a therapeutic target of gene therapy in ES tumors. Since the molecular mechanism of CD99 type II producing these effects remains largely unknown, additional studies are to contribute to elucidate the signaling events of CD99.

\section{Acknowledgment}

This study was supported by a research fund from Korea Science and Engineering Foundation (98-04030201-3).

\section{References}

Alberti I, Bernard G, Alexandre K, Pelassy C, Pourtein M, Aussel C, Bernard A. CD99 isoform expression dictates $T$ cell functional outcomes. FASEB J 2002;10:2-49

Ambros IM, Ambros PF, Strehi S, Kovar H, Gadner H, Salzer $\mathrm{KM}$. MIC2 is a specific marker for Ewing's sarcoma and peripheral primitive neuroectodermal tumors. Evidence for a common histogenesis of Ewing's sarcoma and peripheral primitive neuroectodermal tumors from MIC2 expression and 
specific chromosome aberration. Cancer 1991:67:1886-93

Aubrit F, Gelin C, Pham D, Raynal B, Bernard A. The biochemical characterization of E2, a $T$ cell surface molecule involved in rosettes. Eur J Immunol 1989;19:1431-6

Banting G, Pym B, Darling SM, Goodfellow PN. The MIC2 gene product: epitope mapping and structure prediction analysis define an integral membrane protein. Mol Immunol 1989; 26:181-8

Bernard A, Aubrit F, Raynal B, Pham D, Boumsell L. A T cell surface molecule different from $C D 2$ is involved in spontaneous rosette formation with erythrocytes. J Immunol 1988;140:1802-7

Bernard G, Zoccola D, Deckert M, Breittmayer JP, Aussel $C$, Bernard A. The E2 molecule (CD99) specifically triggers homotypic aggregation of $\mathrm{CD}^{+} \mathrm{CD}^{+}$thymocytes. $\mathrm{J}$ Immunol 1995;154:26-32

Bernard G, Breittmayer JP, Matteis M, Trampont P, Hofpont $P$, Hofman P, Senik A, Bernard A. Apoptosis of immature thymocytes mediated by E2/CD99. J Immunol 1997;158: 2543-50

Bernard G, Bernard A, Picci P. CD99 engagement: an effective therapeutic strategy for Ewing tumors. Cancer Res 2000; 60:5134-42

Cavazzana AO, Miser JS, Jefferson J, Triche TJ. Experimental evidence for a neural origin of Ewing's sarcoma. Am J Pathol 1987;127:507-28

Choi EY, Park WS, Jung KC, Kim SH, Kim YY, Lee WJ, Park SH. Engagement of CD99 induces up-regulation of TCR and MHC class I and class II molecules on the surface of human thymocytes. J Immunol 1998;161:749-54

Choi YL, Chi JG, Suh YL. CD99 immunoreactivity in ependymoma. Appl Immunohistochem Mol Morphol 2001;9:125-9

Devoe K, Weidner N. Immunohistochemistry of small roundcell tumors. Semin Diagn Pathol 2000;17:216-24

Dworzak MN, Fritsch G, Buchinger P, Fleischer C, Printz D, Zellner A, Schollhammer A, Steiner G, Ambros PF, Gadner $\mathrm{H}$. Flow cytometric assessment of human MIC2 expression in bone marrow, thymus, and peripheral blood. Blood 1994;83:415-25

Ellis NA, Ye TZ, Patton S, German J, Goodfellow PN, Weller $P$. Cloning of PBDX, an MIC2-related gene that spans the pseudoautosomal boundary on chromosome Xp. Nat Genet 1994;6:394-400

Ewing J. Diffuse endothelioma of bone. Proc N Y Pathol Soc 1921;21:17-24

Fellinger EJ, Garin CP, Triche TJ, Huvos AG, Retting WJ. Immunohistochemical analysis of Ewing's sarcoma cell surface antigen p30/32 ${ }^{\mathrm{MIC} 2}$. Am J Pathol 1991;139:317-25

Gelin CF, Aurit F, Phalipon A, Raynal B, Cole S, Kaczorek $\mathrm{M}$, Bernard A. The E2 antigen, 32kDa glycoprotein involved in $T$ cell adhesion process, is the MIC 2 gene product. EMBO J 1989;8:3253-9

Gil MC, Lee MH, Seo JI, Choi YL, Kim MK, Jung KC, Park $\mathrm{SH}, \mathrm{Kim}$ TJ. Characterization and epitope mapping of two monoclonal antibodies against human CD99. Exp Mol Med

\section{$2002 ; 34: 411-8$}

Goodfellow PJ, Darling SM, Thomas NS, Goodfellow PN. A pseuautosomal gene in man. Science 1986;234:740-3 Guiter GE, Gamboni MM, Zakowski MF. The cytology of extraskeletal Ewing sarcoma. Cancer 1999;87:141-8

Hahn JH, Kim MK, Choi EY, Kim SH, Sohn HW, Ham DI, Chung DH, Kim TJ, Lee WJ, Park CK, Ree HJ, Park SH. CD99 regulates the LFA-1/ICAM-1-mediated adhesion of lymphocytes, and its gene encodes both positive and negative regulators of cellular adhesion. J Immunol 1997;159: 2250-8

Hara S, Adachi Y, Kaneko Y, Fujizmoto J, Hata J. Evidence for heterogeneous group of neuronal differentiation in Ewing's sarcoma. Br J Cancer 1991;64:1025-30

Hess E, Cohen C, Derose P, Yost B. Nonspecificity of p30/ $32 \mathrm{MIC} 2$ immunolocalization with the 013 monoclonal antibody in the diagnosis of Ewing's sarcoma: application of an algorithmic immunohistochemical analysis. App Immuno Mol Mor 1997;5:94-103

Kim MK, Choi YL, Kim MK, Kim SH, Choi EY, Park WS, Bae YM, Woo SK, Park SH. MHC class II engagement inhibits CD99-induced apoptosis and up-regulation of $\mathrm{T}$ cell receptor and $\mathrm{MHC}$ molecules in human thymocytes and $\mathrm{T}$ cell line. FEBS Lett 2003;546:379-84

Kodama K, Doi O, Higashiyama M, Yokuchi H, Mori Y. Differentiation of Ewing's sarcoma cell line towards neuronal and mesenchymal cell lineages. Jpn J Cancer Res 1994;85: 335-8

Kovar H, Dworzak M, Strehl S, Schnell E, Ambros IM, Ambros PF, Gadner $H$. Overexpression of the pseudoautosomal gene MIC2 in Ewing's sarcoma and peripheral primitive neuroectodermal tumor. Oncogene 1990;45:1067-70

Lee HJ, Kim ES, Jee BK, Hahn JH, Han KH, Jung KC, Park $\mathrm{SH}$, Lee HS. Functional involvement of src and focal adhesion kinase in a CD99 splice variant-induced motility of human breast cancer cells. Exp Mol Med 2002;34:177-83

Moll R, Lee I, Gould VE, Berndt R, Roessner A, Franke WW. Immunocytochemical analysis of Ewing's tumors. Am J Pathol 1987;127:288-304

Noguera R, Triche TJ, Navarro S, Tsokos M. Danamic model of differentiation in Ewing's sarcoma cell lines. Lab Invest 1992;62:143-51

Pettersen RD, Bernard G, Olafsen MK, Pourtein M, Lie SO. CD99 signals caspase-independent $\mathrm{T}$ cell death. J Immunol 2001;166:4931-42

Scotlandi K, Baldini N, Cerisano V, Manara MC, Benini S, Serra M, Nanni P, Nicoletti G, Sohn Scotlandi K, Serra M, Manara MC, Benini S, Sarti M, Maurici D, Lollini PL, Picci $\mathrm{P}$, Bertoni $\mathrm{F}$, Baldini $\mathrm{N}$. Immunostaining of the p30/32MIC2 antigen and molecular detection of EWS rearrangements for the diagnosis of Ewing's sarcoma and peripheral neuroectodermal tumor. Hum Pathol 1996;27:408-16

Shin YK, Lee GK, Kook MC, Jung KC, Kim JR, Song HG, Park SH, Chi JG. Reduced expression of CD99 and functional disturbance in anencephalic cortical thymocytes. Virchows Arch 1999;434:443-9

Sohn HW, Choi EY, Kim SH, Lee IS, Chung DH, Sung UA, 
Hwang DH, Cho SS, Jun BH, Jang JJ, Chi JG, Park SH. Engagement of CD99 induces apoptosis through a calcineurin-independent pathway in Ewing's sarcoma. Am J Pathol 1998;153:1937-45

Tetsuro S, Akihiro $U$, Junichi $H$. Neurogenic potential of Ewing's sarcoma cells. Virchows Arch 1997;430:41-6
Waclavicek M, Majdic O, Stulnig T, Berger M, Sunder PR, Zlabinger GJ, Baumruker T, Stockl J, Ebner C, Knapp W, Pickl WF. CD99 engagement on human peripheral blood T cells results in TCR/CD3-dependent cellular activation and allows for Th1-restricted cytokine production. J Immunol 1998;161:4671-8 